\section{Comparison of the ETDRS logMAR, 'compact reduced logMar' and Snellen charts in routine clinical practice}

L-A Lim', NA Frost ${ }^{2}$, RJ Powell ${ }^{3}$ and P Hewson ${ }^{4}$

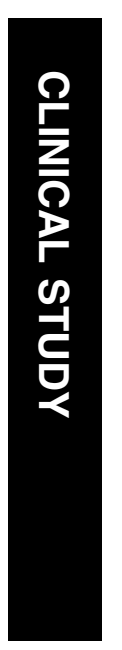

used vision test in clinical practice. The limitations of visual acuity testing need to be well understood.

The most commonly employed visual acuity chart in routine clinical practice is the Snellen chart, following its introduction in 1862.

However the characteristics of the Snellen chart and related charts have been criticised. The scale is not truly interval in nature and different numbers of letters on each line may lead to different legibility due to crowding effects. ${ }^{1}$ These problems have led to the development of alternative charts. ${ }^{2,3}$ Such charts are now standard in research but have not been well adopted in clinical practice, possibly due to the unfamiliar scoring system and the cost in terms of the extra time taken to perform the test. Furthermore, in order to obtain the best performance from the chart a forced-choice method of testing is recommended. ${ }^{4}$ This adds further time to the procedure and is a potential barrier to introduction of $\log$ MAR charts into routine clinical practice. If a forced choice procedure is not adopted it is uncertain whether the benefits of the improved chart design will be of sufficient magnitude to justify their use.

Rosser and Laidlaw developed a 'Reduced $\log$ MAR' (RLM) visual acuity chart designed on the same principles as the ETDRS logMAR chart in terms of the logarithmic progression in letter size, inter-letter and inter-line spacing and use of Sloan set of letters but with a reduction in the number of letters per line. The RLM chart offered improved test-retest variability with a $95 \%$ tolerance limit for change of \pm 0.24 $\log$ MAR, compared to $\pm 0.33 \log$ MAR for the Snellen line assignment method and half the reading time of either the Snellen or the ETDRS $\log \mathrm{MAR}$ charts. Its test-retest variability, however, was not different from the known preferred letter-by-letter scoring method when
${ }^{1}$ The West of England Eye Unit, Royal Devon and Exeter Foundation Trust, Exeter, Devon, UK

${ }^{2}$ Ophthalmology Department, South Devon Healthcare NHS Trust, Torbay Hospital, Lawes Bridge, Torquay, Devon, UK

${ }^{3}$ Research Design Service South West, Royal Devon and Exeter Hospital, Exeter, UK

${ }^{4}$ School of Mathematics and Statistics, University of Plymouth, Drake Circus, Plymouth, UK

Correspondence: L-A Lim, Ophthalmology Department, Royal Devon and Exeter Foundation Trust, The West of England Eye Unit,

Barrack Road,

Exeter,

Devon EX2 5DW,

UK

Tel: +01392411 611;

Fax: +01392 406022 .

E-mail: leiailim@

doctors.org.uk

Received: 19 November 2008

Accepted in revised form: 18 May 2009 Published online: 26 June 2009 
Table 1 Chart design summary

\begin{tabular}{lccccc}
\hline CHART & $\begin{array}{c}\text { No of letters per } \\
\text { line }\end{array}$ & $\begin{array}{c}\text { Line interval in } \\
\text { logMAR }\end{array}$ & $\begin{array}{c}\text { Single letter value } \\
\text { in logMAR }\end{array}$ & $\begin{array}{c}\text { Total number of } \\
\text { letters }\end{array}$ & Acuity range (logMAR) \\
\hline ETDRS & 5 & 0.1 & 0.02 & 70 & +1.08 to -0.30 (4 metres) \\
cRLM & 3 & 0.1 & 0.033 & 42 & +1.07 to $-0.30(4$ metres $)$ \\
Snellen & $1-8$ & $0.08-0.22$ & $0.01-0.11$ & 45 & +1.0 to -0.18 (6 metres) \\
\hline
\end{tabular}

using the Snellen chart, with a 95\% tolerance limit for change of $\pm 0.24 \log$ MAR. ${ }^{5}$

Laidlaw et al then introduced a ' compact Reduced $\log$ MAR' (cRLM) chart, a development of the RLM chart which is closer spaced than the RLM charts and demonstrated a $95 \%$ tolerance limit for change of \pm 0.17 $\log$ MAR in amblyopic children. ${ }^{6}$

This study aimed to compare the performance of ETDRS, 'compact reduced logMAR' and Snellen charts in an ophthalmic outpatient setting. The characteristics of the charts are summarised in Table 1.

\section{Method}

The study was approved by the South Devon Research Ethics Committee. A test-retest study was performed. Each subject made two separate visits to the eye clinic for repeated measurements. One eye per patient was tested.

\section{Subjects}

40 adults were recruited from the outpatient clinics of the Ophthalmology Department. The sample size was estimated using computer simulation with the formula given by Bland and Altman $1^{7}$ based on the anticipated level of agreement in the Laidlaw et al studies.

The Bland and Altman formula required the solution of:

$$
1.96(\mathrm{SD} /(\operatorname{sqrt}(2 n(m-1)))=\mathrm{aSD}
$$

Where $\mathrm{SD}=$ within subject standard deviation, ' $n$ ' $=$ number of patients, ' $m$ ' $=$ number of observations on each patient and ' $a$ ' = agreement limit. The agreement limit was set at $\pm 0.20 \log$ MAR.

The sample was stratified such that 5 patients of each acuity level of the Snellen visual acuity chart were recruited. These patients had stable eye conditions eg diagnosis of cataract, pseudophakia, stable glaucoma, non-exudative age-related macular degeneration and chronic scarring of the cornea. Only subjects who were literate and able to comprehend and perform the test were included. These subjects had variable experience with regards to chart reading and no practice runs were carried out prior to them reading the charts for the study.

\section{The charts and display}

Two versions of the cRLM chart were loaned from Rosser and Laidlaw and two versions of the ETDRS logMAR chart were displayed in the standard Lighthouse light box. Two versions of the Snellen chart were displayed in the standard Clement Clark light box. All charts were backlit in their respective light box.

The charts were viewed under the same lighting conditions as the normal eye clinic.

\section{The testing protocol}

The subjects wore their habitual spectacle correction. The visual acuity was tested with the subject seated at 6 metres for the Snellen chart and at 4 metres for the ETDRS and the cRLM charts. Subjects were randomised to one of two versions of the aforementioned charts. The order of presentation of the charts was also randomised at each visit. An online randomiser was used to generate 80 sets of numbers dictating the version and the order of chart read. The same eye was tested for all the charts at both visits. The interval between the two tests ranged from 1-3 weeks.

A single examiner was employed. The subject was requested to start at the top left of each chart, read from left to right and work their way down the chart. The subjects were instructed to give one reading for each letter, guessing if they were unsure. They were asked once to guess when they reached lines where they could not see clearly. The subjects were timed from the start of reading the chart to when all the letters had been attempted or when they pronounced they could guess no further after encouragement. In order to mimic the clinical setting, forced-choice testing (which would have required the patients to guess a complete line incorrectly) was not used.

The examiner recorded the results on prepared proformas so that calculations could be done separately to avoid influencing the measurement time.

\section{Scoring}

All charts were scored for individual letters. All calculations were performed using logMAR values. The 
value of the letters on the Snellen chart was converted to $\log$ MAR format as follows:

The line acuity was obtained by taking the log to the base 10 of the reciprocal of the Snellen acuity fraction. The logMAR value of an individual Snellen letter on a particular line was calculated by subtracting the logMAR value of the line above from the $\log$ MAR value of that particular line divided by the number of letters on the particular line. (The same method is employed routinely for the ETDRS Chart)

Each letter on the ETDRS logMAR and the cRLM charts were scored in keeping with the principle of scoring the ETDRS chart and the cRLM chart.

\section{Analysis}

The results were examined initially by inspecting Bland and Altman plots.

The possibility of systematic bias between charts was examined by comparing the median acuity values of the charts from the first round of testing, using Kruskal-Wallis Test.

The possibility of a practice or learning effect was examined by comparing the median acuities on test and retest, using Wilcoxon's signed ranks test.

Test-retest variability was examined by calculating the differences between 'test' and 'retest'. The 95\% tolerance limits for change (95\% TLC) was calculated as 1.96 times the standard deviation of the differences found on 'test' and 'retest'.

The reading speeds from the first round of testing were compared using a paired $t$-test and ANOVA.

\section{Results}

19 males and 21 females were recruited. The ages ranged from 35 to 92 with mean age of 67 and median age of 64 years. The diagnosis included cataract, glaucoma, ocular hypertension, age-related macular degeneration, epiretinal membrane and corneal scar from previous herpetic keratitis.

The range of visual acuities recorded were -0.10 to 1.06 for the ETDRS, -0.13 to 1.07 for the cRLM and -0.10 to 1 for the Snellen charts. The median acuity values of the ETDRS, cRLM and Snellen charts were 0.42, 0.41 and 0.41 respectively. Statistically, there were no significant differences between the three different charts in terms of median acuity ( $P=0.9865$ by Kruskal - Wallis Test).

In all three chart types, a small practice effect was observed, the visual acuity improving on retesting. This was significant for the Snellen chart $(P<0.0001)$ but not so for the ETDRS chart $(P=0.1976)$ or cRLM chart $(P=0.2098)$ by Wilcoxon's signed ranks test). (Table 2$)$

The reliability in terms of the $95 \%$ tolerance limits for change was the best for the ETDRS chart and poorest for the Snellen chart. However the difference in performance was fairly small, amounting to $0.04 \log$ MAR or 2 ETDRS letters.

The mean and range of testing times were: ETDRS 34.65 (9.38-80.18) seconds, cRLM 21.17 (5-40) seconds, Snellen 18.67 (3-35.56) seconds. Using ANOVA, this difference between testing times was highly significant $(P<0.0001)$.

\section{Discussion}

In our study, with the exception of testing times, the performance of the three charts was fairly similar. In Laidlaw et al's study in amblyopic children, the differences in reliability were greater, with $95 \%$ tolerance limits for change of \pm 0.14 (7 ETDRS letters) for ETDRS logMAR chart, \pm 0.17 (5 cRLM letters) for the compact reduced $\log$ MAR chart, and \pm 0.29 for the Snellen chart (variable number of Snellen letters). ${ }^{6}$

Gibson and Sanderson ${ }^{8}$ reported up to $13 \%$ subjects displaying discrepancies of 2 lines or more and only $1 / 3$ subjects with the same visual acuity on the Snellen chart on repeated testing, suggesting that changes in acuity of 2 lines or less on the Snellen chart may not be clinically significant. This study was performed by two investigators and therefore the results may have been influenced by interobserver variability as well as the use of the line assignment method.

Arditi et $a l^{9}$ and Bailey et $a l^{10}$ demonstrated reliability of the ETDRS logMAR chart to be within $\pm 0.10 \log$ units (5 letters). The former employed strict laboratory conditions with 5 highly practised subjects with normal visual acuity and the latter used 21 normal sighted

Table 2 Performance data for the charts

\begin{tabular}{lccccccc}
\hline & $\begin{array}{c}\text { Median difference } \\
\text { in logMAR }\end{array}$ & $\begin{array}{c}\text { P-value for } \\
\text { median difference }\end{array}$ & $\begin{array}{c}\text { 95\% tolerance } \\
\text { limits for change }\end{array}$ & $\begin{array}{c}\text { Upper } \\
\text { limit }\end{array}$ & $\begin{array}{c}\text { 95\% C.I for } \\
\text { upper limit }\end{array}$ & $\begin{array}{c}\text { Lower } \\
\text { limit }\end{array}$ & $\begin{array}{c}\text { 95\% C.I for } \\
\text { lower limit }\end{array}$ \\
\hline ETDRS1-ETDRS2 & 0.01 & 0.2 & \pm 0.14 & 0.15 & $0.10,0.19$ & -0.13 & $-0.17,-0.08$ \\
cRLM1-cRLM2 & 0.02 & 0.2 & \pm 0.16 & 0.18 & $0.13,0.23$ & -0.14 & $-0.20,-0.09$ \\
Snellen1- Snellen2 & 0.05 & $<0.0001$ & \pm 0.18 & 0.23 & $0.18,0.30$ & -0.13 & $-0.18,-0.07$ \\
\hline
\end{tabular}


subjects at 3 different testing distances. Elliot and Sheridan ${ }^{11}$ found the $95 \%$ confidence limits for retest variability to be 3.5 letters, this was done with 21 subjects reading the ETDRS chart 3 times on 2 occasions, 1 week apart. Charlotte et $a l^{12}$ compared 4 different logMAR charts and found the reliability to range from \pm 0.10 to \pm 0.16 . Siderov et al $^{16}$ attempted to simulate variability of visual acuity measurement in a large eye clinic by incorporating up to 21 different examiners with variable chart design features and reported reliability of \pm 0.15 . Rosser et $\mathrm{l}^{14}$ concluded that the ETDRS chart can reliably detect a change of $0.2 \log$ MAR (with a sensitivity of $100 \%$, $95 \%$ C.I $93-100 \%$ and specificity of $96 \%, 95 \%$ C.I. 86-99.5\%), but not an acuity change of $0.10 \log$ MAR (sensitivity $38 \%, 95 \%$ C.I. $25-53 \%$ ). The latter attempted to simulate acuity change by varying the test distance at which 3 versions of ETDRS charts were read under experimental conditions. However, as mentioned above the testing conditions and subjects may have been different to our study, giving an impression of the advantages of logMAR charts which may not be achievable in clinical practice.

Although the theoretical limitations of the Snellen chart and the advantages of logMAR charts are wellrehearsed $^{5,6,8-12}$ and demonstrable in research settings it is less certain as to whether the theoretical benefits will be realised in routine clinical practice. Moreover, the performance of a visual acuity test chart is dependent on the difficulty score of the letter as well as line. Line difficulty score depends on the number of letters. The number of letters in the Snellen chart varies from 1 at the low visual acuity range to 8 at the higher visual acuity ranges. This is likely to make the Snellen chart better than others at a higher visual acuity ranges and worse at the lower acuity ranges. The rationale behind stratifying the sample collection in this study was to ensure equal representation of each level of the Snellen acuity range.

The method of scoring individual letters is superior to the line assignment method. ${ }^{9,10,13}$ However it is not known whether letter-by letter scoring is used consistently in ophthalmic clinics.

Comparison between charts revealed no systematic bias in terms of the mean acuity measurements and only small differences in test-retest reliability, but the ETDRS $\log$ MAR chart took nearly twice the amount of time to complete compared to the other charts. Therefore the present study would suggest that, based on the method employed, the introduction of routine use of the ETDRS chart may not be cost-effective. Camparini et $a l^{15}$ advocated a psychophysical adaptive method 'ETDRSFast' of measuring visual acuity, whereby subjects were required to identify only one letter per line, and at the first letter incorrectly read, the subject was required to read the whole of the preceding line. They reported a significant reduction of test time by $30 \%$ and a better reliability of \pm 0.06 (3 letters) compared with \pm 0.08 (4 letters) using the standard method. This method could be introduced to reduce the time taken to read the test charts further in the eye clinic but is not currently routinely used. Another method to reduce chart reading time would be 'bracketing' where the subject was asked to read the smallest line they could see on the chart then encouraged to read further until threshold was reached.

It is possible that the reliability of testing in clinical practice could be improved by adopting a forced-choice testing protocol, thus moving closer to the methods employed in research studies. However this method is even more time-consuming and would need to be used consistently by all clinic personnel.

In making a decision about chart selection other issues also need to be taken into account. The relative lack of Snellen letters per row in the low-acuity range suggests a greater role for the EDTRS chart in Low vision testing. The advantages of ETDRS in terms of reliability may have a greater role in Low vision patients but this would need to be verified with appropriately designed studies. Adjustment for testing distance is possible with the Snellen chart, but to make full use of the letter-by-letter scoring method, complex calculations are needed. The time needed for these calculations would need to be added to the testing time. However as electronic patient records are introduced, the calculations could be automated. Use of the compact reduced $\log$ MAR would improve the ease of scoring, compared to Snellen, without increased testing time but with little added advantage in terms of reliability. A decision to upgrade a clinic would of course also include equipment costs and staff training costs.

In summary, the theoretical advantages of $\log$ MAR charts compared to Snellen charts are measurable in a simulated clinical setting but the magnitude of the benefit of using an improved chart design appears to be small and the cost-effectiveness of introducing such charts into routine clinical practice is uncertain.

\section{Acknowledgements}

Dr Roy Powell, Consultant, Research Design Service South West and Research Fellow, Peninsula School of Medicine \& Dentistry; Paul Hewson, Lecturer in Statistics, University of Plymouth for their statistical input into this study.

Funding: Funded by the Department of Ophthalmology, South Devon Healthcare NHS Trust, Torbay Hospital, Torquay, TQ2 7AA

\section{Conflict of interest}

The authors declare no conflict of interest. 


\section{References}

1 McGraw P, Winn B, Whitaker D. Reliability of the Snellen chart. BMJ 1995; 310: 1481-1482.

2 Bailey IL, Lovie JE. New Design Principles for visual acuity letter charts. Am J Optom Physiol Opt 1976; 53: 740-745.

3 Ferris III FL, Kassoff A, Bresnick GH, Bailey I. New visual acuity charts for clinical research. Am J Ophthalmol 1982; 94: 91-96.

4 Carkeet A. Modeling logMAR visual acuity scores: effects of termination rules and alternative forced-choice options. Optom Vis Sci 2001; 78: 529-538.

5 Rosser DA, Laidlaw DAH, Murdoch IE. The development of a 'reduced $\log M A R^{\prime}$ visual acuity chart for use in routine clinical practice. Br J Ophthalmol 2001; 85: 432-436.

6 Laidlaw DA, Abbott A, Rosser DA. Development of a clinically feasible logMAR alternative to the Snellen chart: performance of the 'compact reduced logMAR' visual acuity chart in amblyopic children. Br J Ophthalmol 2003; 87: 1232-1234.

7 Bland J M, Altman DG. Statistics Notes: Measurement error? BMJ 1996; 313: 744.

8 Gibson SA, Sanderson HF. Observer variation in ophthalmology. Br J Ophthalmol 1980; 64: 457-460.
9 Arditi A, Cagenello R. On the statistical reliability of letter chart visual acuity measurements. Invest Ophthalmol Vis Sci 1993; 34: 120-129.

10 Bailey IL, Bullimore MA, Raash TW, Taylor HR. Clinical grading and the effects of scaling. Invest Ophthalmol Vis Sci 1991; 32: 422-432.

11 Elliot DB, Sheridon M. The use of accurate visual acuity measurements in clinical anti-cataract formulation trials. Ophthalmic Physiol Opt 1988; 8: 397-401.

12 Hazel CA, Elliott DB. The Dependency of LogMAR Visual Acuity Measurements on Chart Design and Scoring Rule. Optometry and Vision Science 2002; 79: 788-792.

13 Vanden Bosch ME, Wall M. Visual acuity scored by the letter-by-letter or probit methods has lower retest variability than the line assignment method. Eye 1997; 11: 411-417.

14 Rosser DA, Cousens SN, Murdoch IE, Fitzke FW, Laidlaw DA. How sensitive to clinical change are ETDRS $\log$ MAR visual acuity measurements? Invest Ophthalmol Vis Sci 2003; 44(8): 3278-3281.

15 Camparini M, Cassinari P, Ferrigno L, Macaluso C. ETDRSFast: Implementing psychophysical adaptive methods to standardized visual acuity measurement with ETDRS charts. Invest Ophthalmol Vis Sci 2001; 42(6): 1226-1231.

16 Siderov J, Tiu AL. Variability of measurements of visual acuity in a large eye clinic. Acta Ophthalmol Scand 1999; 77: 673-676. 